\title{
RESEARCH
}

\section{Two successive outbreaks of mumps in Nova Scotia among vaccinated adolescents and young adults}

\author{
Gaynor Watson-Creed, Andrea Saunders, Jeffrey Scott, Luis Lowe, Janice Pettipas, Todd F. Hatchette
}

$\infty$

See related article page 49I

\section{ABSTRACT}

Background: Before the widespread use of vaccine, mumps was the most common cause of viral meningitis (up to $10 \%$ of mumps infections). Vaccination programs have resulted in a drop of more than $99 \%$ in the number of reported mumps cases in the United States and Canada. Although rare in Canada, outbreaks have recently occurred throughout the world, including a large outbreak in the United Kingdom, where more than 56000 cases were reported in 2004-2005.

Methods: Two recent outbreaks in Nova Scotia were investigated by public health officials. Cases were defined by laboratory confirmation of infection (i.e., isolation of mumps virus by culture) or clinical diagnosis in people epidemiologically linked to a laboratory-confirmed case. The people infected were interviewed to determine possible links and to identify contacts. Mumps virus was cultured from urine and throat specimens, identified via reverse-transcriptase polymerase chain reaction (RT-PCR) and subjected to phylogenetic analysis to identify the origin of the strain.

Results: The first outbreak involved 13 high-school students (median age $14 \mathrm{yr}$ ): 9 who had previously received 2 doses of measles-mumps-rubella vaccine (MMR) and 4 who received a single dose. The second outbreak comprised 19 cases of mumps among students and some staff at a local university (median age $23 \mathrm{yr}$ ), of whom 18 had received only 1 dose of MMR (the other received a second dose). The viruses identified in the outbreaks were phylogenetically similar and belonged to a genotype commonly reported in the UK. The virus from the second outbreak is identical to the strain currently circulating in the UK and United States.

Interpretation: The predominance in these outbreaks of infected people of university age not only highlights an environment with potential for increased transmission but also raises questions about the efficacy of the MMR vaccine. The people affected may represent a "lost cohort" who do not have immunity from natural mumps infection and were not offered a 2-dose schedule. Given the current level of mumps activity around the world, clinicians should remain vigilant for symptoms of mumps.

CMAJ 2006;175(5):483-8
$M$ umps is a highly infectious, vaccine-preventable disease that is endemic worldwide. The virus spreads easily via respiratory droplets, typically causing acute parotitis, although $15 \%-20 \%$ of mumps infections can be asymptomatic and 50\% can be associated with nonspecific or respiratory symptoms..$^{1,2}$ Complications can include pancreatitis, orchitis, meningitis and encephalitis. ${ }^{3}$ Routine vaccination has resulted in a dramatic drop in the number of reported mumps cases in the United States and Canada since the vaccine was licensed in $1969 .{ }^{4,5}$ Although rare in Canada, outbreaks have recently occurred elsewhere, including a large outbreak of mumps in the United Kingdom, where more than 56000 cases were reported in 2004-2005. ${ }^{6}$

Measles-mumps-rubella (MMR) vaccine was introduced in Nova Scotia in 1975 as a single dose to children aged $12-15$ months. In 1996 , a second dose was added for children aged 4-6 years as part of a national strategy against measles. ${ }^{7}$ Since then, fewer than 2 cases of mumps have been reported annually in Nova Scotia, most of which occurred among people who were never immunized. ${ }^{8}$

On August 2, 2005, 2 cultures positive for mumps were reported to public health officials in the Capital District Health Authority in Halifax, NS. The isolates were obtained from 2 previously vaccinated adolescent girls who attended the same junior high school. When a second cluster of infections was identified in September of that year among staff and students of a large university in Halifax, possible links between the 2 outbreaks were investigated. Virus genotyping aided in the identification of the sources of the outbreaks. In this report we describe the epidemiologic and laboratory findings from these outbreaks and examine the potential public-health implications of mumps in Canada.

\section{Methods}

Cases were categorized according to the definitions used for national surveillance. ${ }^{9}$ A case was considered confirmed if it involved laboratory confirmation of infection (i.e., isolation of the mumps virus by culture) or was a clinical diagnosis in someone who was epidemiologically linked to a case in which infection was laboratory-confirmed. Identification of mumps genome by reverse-transcriptase polymerase chain reaction (RT-PCR) from a throat swab or urine sample was also used. A case was considered probable if someone with clinical ill- 
ness was diagnosed in the absence of appropriate laboratory tests and had no known epidemiological link to a laboratoryconfirmed case. Clinical illness was characterized by acute onset of unilateral or bilateral tender, self-limited swelling of the parotid or other salivary gland, lasting 2 or more days, and without other apparent cause. ${ }^{9}$

The people infected were interviewed to obtain their demographic and clinical information and vaccination status. Details were sought on their activities during the communicable period (i.e., from 7 days before until 9 days after symptom onset) to compile names of potential contacts for follow-up. Similar information was recorded for the exposure period (I4-25 days before symptom onset) in order to determine possible epidemiological links to other confirmed or probable cases. ${ }^{10}$

To manage the outbreaks, we asked people with mumps infection to isolate themselves until 9 days after the onset of symptoms, avoid infecting others. Their household members and other close contacts were advised to seek an additional MMR vaccination from their health care provider if they had no history of infection with mumps and had previously received only one dose of MMR vaccine or were unvaccinated.

Urine and throat cultures were inoculated onto primary monkey-kidney and vero cell lines and monitored daily for cytopathic effect (CPE). To identify the presence of virus, we used an indirect immunofluorescence assay (Chemicon [Millipore], Temecula, Calif.) at the end of the 7-day incubation period or as soon as CPE was noted. Viral RNA was extracted from throat and urine samples by means of the QIAamp Viral RNA Mini kit (Qiagen, Valencia, Calif.). For genotyping, RTPCR was performed with a heminested protocol specific for the short hydrophobic (SH) gene. ${ }^{11}$ Amplicons were resolved by agarose gel electrophoresis and purified with the QIAquick gel extraction kit (Qiagen). Amplicons were sequenced at the DalGEN Microbial Genomics Centre (Dalhousie University, Halifax, NS) via a Beckman CEQ 8000 capillary sequencer and compared with reference strains from GenBank. DNA sequences were aligned by use of the Lasergene sequence-analysis software package (DNAStar, Madison,
Wis.). We generated phylogenetic trees using parsimony algorithms and bootstrap analysis in Megalign (DNAStar) and Phylogenetic Analysis Using Parsimony software (PAUP 4.o b10, Sinauer Associates, Inc., Sunderland, Mass.).

\section{Results}

\section{Epidemiologic investigation}

Between May and August 2005 (outbreak 1), a total of I3 cases of mumps were linked to the outbreak either by laboratory confirmation (3 cases, by viral culture) or epidemiologically. The median age of those infected was I4 years (range I3I9 $\mathrm{yr}) ; 8(62 \%)$ were girls. All I 3 had unilateral or bilateral parotitis; 4 (3I\%) also developed fever. No other sequelae of mumps were reported. Nine of the teenagers $(69 \%)$ had received 2 doses of MMR vaccine; 4 (3I\%) had received only 1 dose (Table I).

The index case for the outbreak was a I9-year-old man. The source of his infection could not be determined. He transmitted the infection to a younger sibling, who passed it to her friends and social contacts. Most of the people infected attended a single junior high school. More than 400 contacts were investigated, both nationally and internationally, but no additional cases were identified.

From September 2005 to January 2006 (outbreak 2), a total of Ig cases of mumps (all confirmed by RT-PCR) were reported among the staff and students of a local university. The median age of those infected was 23 years (range 20-27 yr); the majority $(60 \%)$ were men. The 17 students affected attended several different faculties at the university. All of those affected had unilateral $(n=\mathrm{I} 2)$ or bilateral $(n=7)$ parotitis; I2 $(63 \%)$ also developed fever; and 1 developed aseptic meningitis (no other complications were reported). In only 1 case had 2 doses of MMR vaccine been received; in the remaining I8 (95\%), only 1 dose of the vaccine had been administered in childhood (Table I). The index case for the second cluster was a local musician who performed at venues regularly attended by university students.

The epidemic curves for both outbreaks are shown in Fig. I. No epidemiologic links between outbreaks 1 and 2 could be discovered.

Two additional laboratory-confirmed cases of mumps identified during the outbreak investigation could not be linked epidemiologically to either cluster. Subsequent phylogenetic analysis showed the mumps virus that was isolated to be distinct from the outbreak strains (Fig. 2).

\section{Microbiologic investigation}

Phylogenetic analysis of the SH gene of the viruses identified during these 2 outbreaks revealed that they differed by only 1 nucleotide from one another, and were related to the genotype $\mathrm{G}$ mumps virus (Fig. 2) that has been circulating in the United Kingdom (Dr. Li Jin,

Note: Pts $=$ patients, Prov. $=$ province,$M M R=$ measles - mumps-rubella $($ vaccine $), N S=$ Nova Scotia 
WHO Global Specialised Laboratory for Measles and Rubella (Mumps), London, UK: personal communication, March 2006), and were clearly distinct from the Jeryl-Lynn strain (genotype A) used in Canadiano vaccine preparation since I988. Two isolates that were identified after the end of the outbreaks also clustered with the outbreak strains: one isolate (Halifax 05) was acquired in the UK; the origin of the other (Halifax o6) is unknown and no links with the outbreak groups could be discovered. Additional information from the National Microbiology Laboratory (Winnipeg, Man.) and the US Centers for Disease Control and Prevention (Atlanta, Ga.) revealed that the Halifax strain identified during the second outbreak was identical to viruses identified in Quebec (Dr. Graham Tipples, Public Health Agency of Canada, Winnipeg, Man.: personal communication, February 2006), New Jersey (DQ66I744) and Iowa (DQ66I745) (Table 2, Fig. 2). The phylogenetic tree further discriminates the outbreak strains from 2 other sporadic cases that appear to be unrelated to the outbreaks: one that was likely acquired in Pakistan (Halifax 03), and another isolate, the origin of which remains unknown (Halifax 04).

\section{Interpretation}

The Halifax outbreaks have occurred in both a doubly vaccinated cohort of adolescents, and a singly vaccinated cohort of young adults. The nature of these outbreaks raises questions as to the efficacy of the MMR vaccine in each of these cohorts. Although the Jeryl-Lynn vaccine strain is thought to induce antibodies in $95 \%$ of people after 1 dose, recent studies $^{5,12,13}$ have estimated the efficacy of a single dose to be less than $70 \%$. It is clear that schedules of 2 doses, such as those used in Canada since r996, have eliminated mumps in some countries, most likely by reducing the rate of primaryvaccine failure. ${ }^{6,14}$

Primary-vaccine failure has been suggested as a cause of our outbreaks and of those now occurring in the US Midwest. However, field studies suggest that reductions in vaccine efficacy likely reflect a combination of both primary and secondary failure of the mumps component of the MMR vaccine. ${ }^{13}$ Unfortunately, lot numbers for the administered vaccines were not available for review during our outbreaks. Improper handling or administration of the vaccines by providers may also have led to some degree of "failure" in these cases.

Central to the development of a vaccine against mumps has been the understanding that, historically, all mumps viruses belong to a single serotype. Vaccination with one strain is thereby thought to prevent subsequent infection by other strains of mumps virus. Mumps has been classified into I2 different phylogenetic clusters called genotypes based on comparison of the $\mathrm{SH}$ gene, the most variable region of its genome. ${ }^{15}$ However, there are data to suggest that the immune response directed against one genotype of mumps may not provide absolute protection against infection with mumps viruses having other genotypes. ${ }^{16}$ There have been no

Outbreak 1 (high-school students), $n=13$

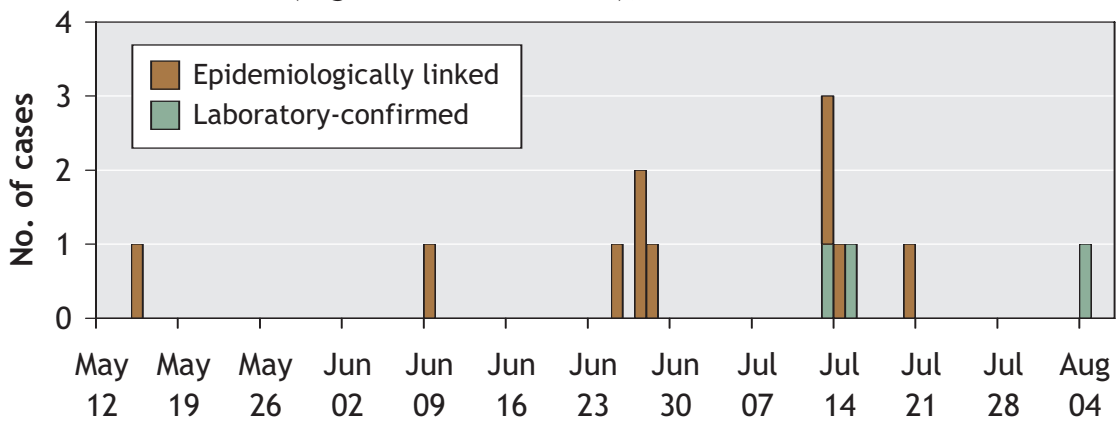

Outbreak 2 (university students and staff), $n=19$

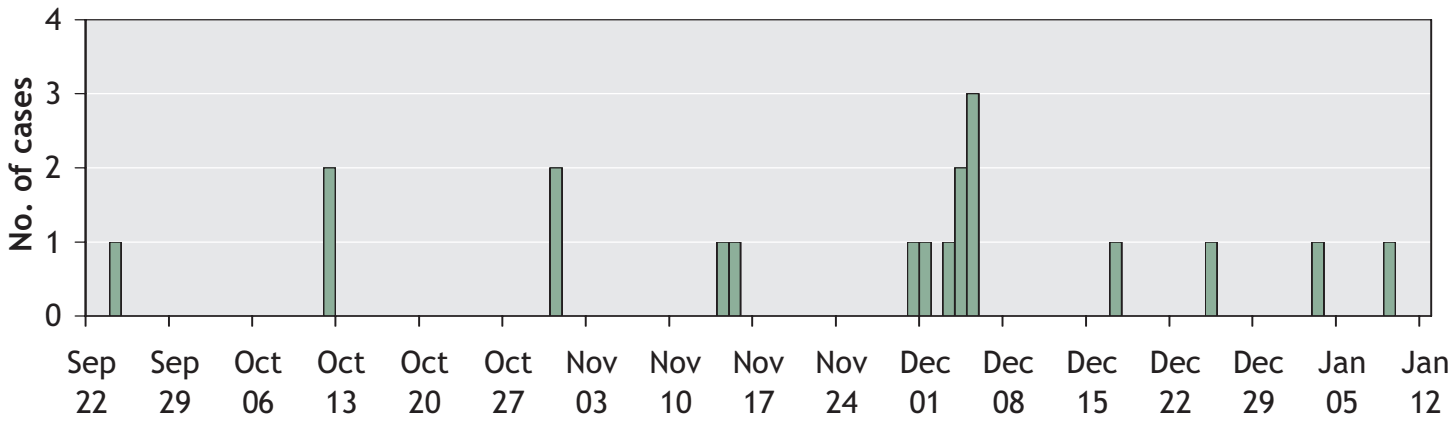

Date of symptom onset, 2005-2006

Fig 1: Cases of mumps in Nova Scotia from May 2005 through January $2006(n=32)$. 
studies examining the vaccine-generated immune response against the genotype identified in our outbreaks.

What greatly facilitated our outbreak investigation was the use of phylogenetic analysis. It allowed us to identify the circulating strain and to differentiate infections by that strain from "sporadic" cases of mumps that were identified during the outbreak period. It also enhanced our understanding of the epidemiology of the disease and its relationship to the UK outbreak.

Recently, an outbreak of mumps at a summer camp in New York was traced back to an unvaccinated camp counsellor from the UK. ${ }^{17}$ Although no isolates from this cluster

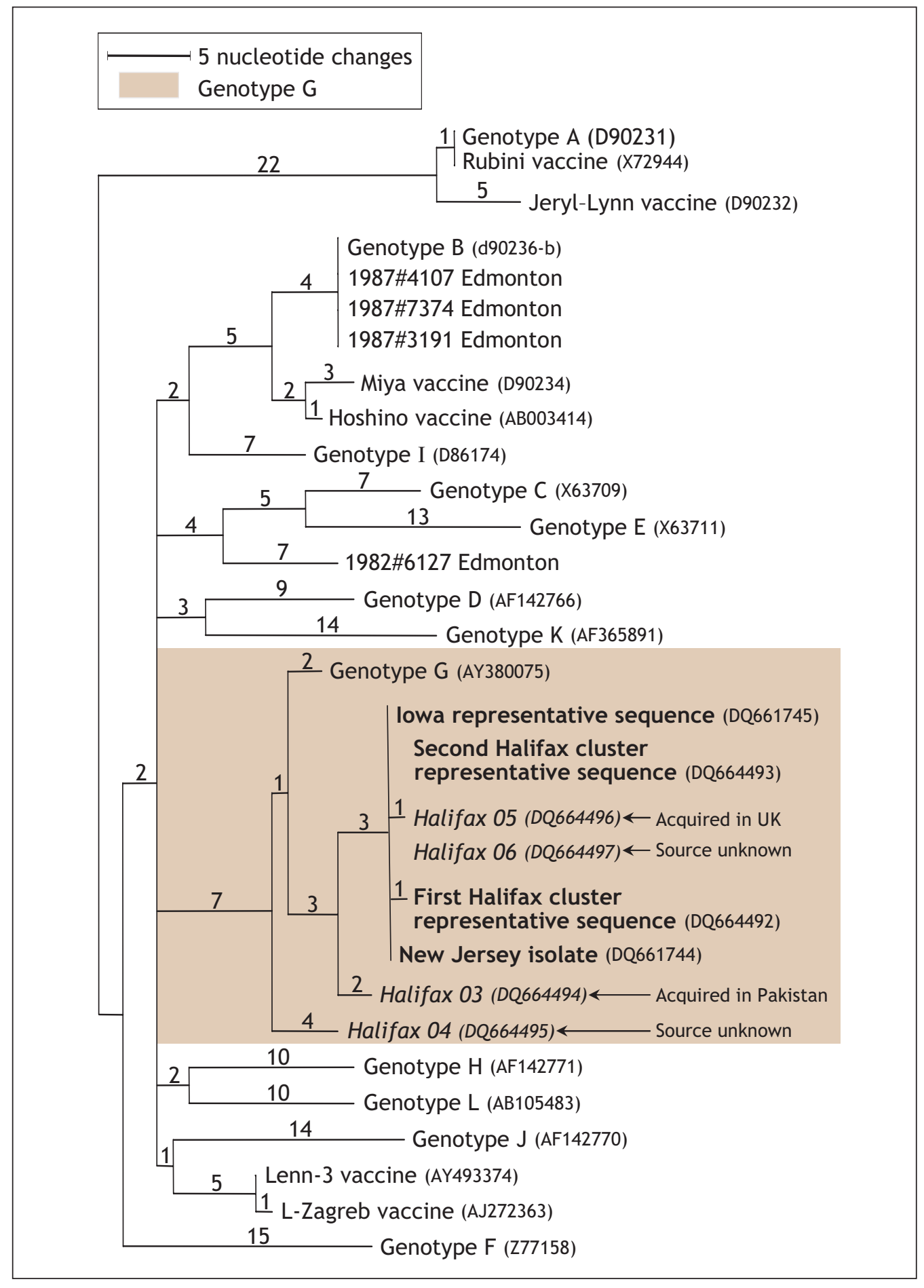

Fig 2: Phylogenetic tree depicting how the viral strains of the Halifax outbreak relate to other mumps viruses previously and currently circulating. Note: codes in parentheses are GenBank numbers. "Source unknown" indicates that no epidemiological links could be found to either outbreak. 
were available for genotyping, an isolate from New Jersey (DQ661744) found during the same period was identical to the Halifax strain identified during outbreak 2. The musician who constituted the index case from outbreak 2 visited New England during his incubation period and may have acquired the infection there. An identical strain (DQ66r745) is now causing the large outbreak of mumps in the Midwestern United States and has also caused mumps in Quebec. Mumps virus genotyping has been done only occasionally in the past in Canada, and this strain has not been previously found (Dr. Graham Tipples: personal communication, February 2006). Although no epidemiologic links between the Halifax outbreak and the US or Quebec cases have been found, the strain is related to genotype $G$ viruses that are currently circulating in the UK (Dr. Li Jin: personal communication, March 2006). Its presence in all 3 places likely represents the concurrent introduction of a dominant mumps virus into North America from the UK.

Methods used to control the outbreak included the tracing of contacts and self-isolation of cases. We used 9 days as the outer limit of the infectious period, compared with the 5-day periods used by others. ${ }^{18}$ Collaboration with the affected university allowed isolations to occur with as little disruption as possible to students.

Although vaccination strategies such as those currently being used to control the US outbreak were considered, no mass immunization campaign or "catch-up" campaign for mumps was undertaken during our outbreaks, for several reasons. ${ }^{19}$ Vaccination levels in both of the age groups affected were already known to be high, and there was no evidence of rapid spread or spread to unvaccinated groups. A clearly favourable cost-benefit ratio could not be shown for conceivable vaccination strategies, particularly in light of UK experience that did not support vaccination as an effective outbreak control strategy in this setting (Drs. Eithne MacMahon, Virology Section, Department of Infection, Guy's and St Thomas' NHS Foundation Trust, and Mary Ramsey, Health Protection Agency Centre for Infections, London, UK: personal communications, January 2006). In our case, an "at risk" population in need of rapid vaccination for outbreak

Table 2: Representative sequences of the mumps viruses described in the study

\begin{tabular}{|c|c|}
\hline Mumps strain & GenBank no. \\
\hline lowa outbreak & DQ661745 \\
\hline New Jersey outbreak & DQ661744 \\
\hline \multicolumn{2}{|l|}{ Cases in Halifax, Nova Scotia } \\
\hline Outbreak 1 (high-school cluster) & DQ664492 \\
\hline Outbreak 2 (university cluster) & DQ664493 \\
\hline \multicolumn{2}{|l|}{ Sporadic cases } \\
\hline Acquired in Pakistan & DQ664494 \\
\hline Acquired in the United Kingdom & DQ664496 \\
\hline Sources unknown; no links to outbreaks & $\begin{array}{l}\text { DQ664495 } \\
\text { DQ664497 }\end{array}$ \\
\hline
\end{tabular}

control could not be easily distinguished from the remainder of the population, especially in outbreak 2 . Several universities are located in the Halifax area, with many students who attend classes on several campuses; it therefore became difficult to identify campuses that were not "at risk". Since resources are limited in Nova Scotia, any decision to revaccinate such a large cohort must be measured against competing public health priorities.

Of note, our outbreak did not spread to the degree that has been seen in the United States, despite the large number of universities in the city. Moreover, very few secondary cases outside of Nova Scotia have been identified, despite travel of the university group during December, the peak of outbreak activity. Together, these findings suggest that a high degree of herd immunity already exists within Nova Scotia and other Canadian populations.

It is unclear as to why outbreaks have occurred in Halifax but not in other regions in Canada, since our history with MMR is likely not much different than that of other provinces. Moreover, many of the cases in outbreak 2 were vaccinated in other provinces (Table I), so the history of the vaccine in Nova Scotia alone provides only a part of the picture. The predominance of people of university age infected during the Halifax and US outbreaks not only highlights a potential environment for increased transmission but may also represent a "lost cohort" that received only a single dose of vaccine in the I970s and I980s, but that did not otherwise have immunity from natural mumps infection and were not offered the improved immunity of a 2 -dose schedule.

The alertness of local physicians in identifying clinical mumps contributed greatly to the successful control of the outbreaks. Mumps should remain on the list of differential diagnoses considered for anyone presenting with parotitis, regardless of his or her vaccination status. Prompt reportage of clinical cases to public health officials, early laboratory confirmation, aggressive tracing of contacts and isolation remain the cornerstones of outbreak management.

The resurgence of mumps in North America underscores the need for a national immunization strategy. Our experience suggests the need for a national discussion of the current and past approaches to mumps. Within that dialogue, strategies for outbreak control must be differentiated from strategies that generally improve herd immunity in the population, such as revaccination campaigns.

This article has been peer reviewed.

From Nova Scotia Health Promotion and Protection (Watson-Creed, Saunders, Scott), the Departments of Community Health and Epidemiology (Watson-Creed, Scott), Pediatrics (Scott) and Pathology (Hatchette), Dalhousie University, and the Department of Pathology and Laboratory Medicine (Pettipas, Hatchette), QEII Health Science Centre, Halifax, NS; the Canadian Field Epidemiology Program (Saunders), Public Health Agency of Canada, Ottawa, Ont.; and the Respiratory and Enteric Virus Branch (Lowe), Centers for Disease Control and Prevention, Atlanta, Ga.

Competing interests: None declared.

Contributors: Gaynor Watson-Creed was responsible for day-to-day management of the outbreak and drafted the Interpretation section of the paper. Andrea Saunders compiled and analyzed the data, coordinatied provincial surveillance efforts and drafted the Results section of the article. Jeff Scott 


\section{CMA}

\section{Back to School Promotion*}

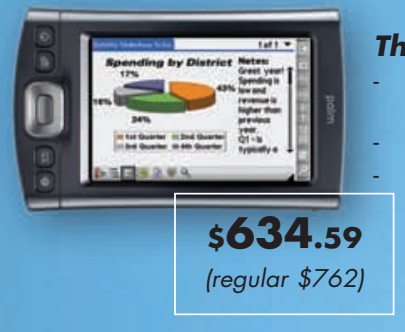

The Ultimate

Palm TX + Universal Wireless Keyboard Lexi-Complete (1 year) Kingston - 1GB SD Memory Card

\section{The Premium}

Palm TX + Universal Wireless Keyboard Lexi-Clinical Suite (1 year) Kingston - 512MB SD Memory Card

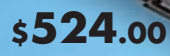
(regular \$613)
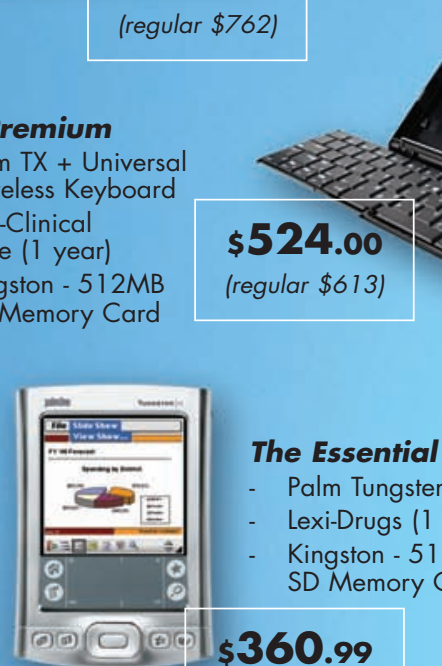

\section{The Essential}

Palm Tungsten E2

Lexi-Drugs ( 1 year)

Kingston - $512 \mathrm{MB}$

SD Memory Card

\section{$\$ 360.99$}

(regular \$415)

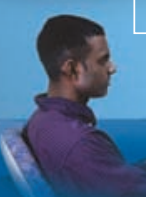

(regular $\$ 215)$

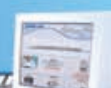

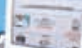

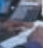

(2)

\section{WIN your purchasel}

Place an order between August 1st and

September 30 th and you could win what you buy! Details online.

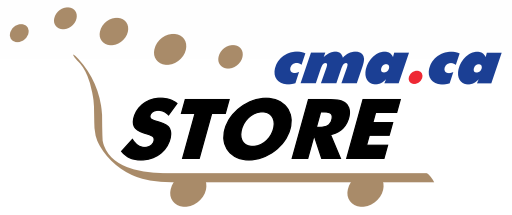

You could win the total amount of your purchase by placing an order in the Online Store between August 1 st and Sept. 30th, 2006. A winner will be selected amongst all the orders received, every two weeks on the following dates: August 21, September 4, September 18, and October 2, 2006. The names of our winners will be posted in our section "@the store" on cma.ca home page. Good luck! *CMA members only. contributed discussions and collaboration with health authorities in other jurisdictions, including the United Kingdom. Luis Lowe coordinated with the laboratory to provide key phylogenetic information and analysis. Janice Pettipas did the day-to-day processing of outbreak-related specimens. Todd Hatchette coordinated the phylogenetic analysis and drafted the Methods and introductory parts of the paper. All 6 coauthors revised the draft manuscript and approved the final version of the paper.

Acknowledgements: We thank several people for data collection, analysis, technical support, advice and comments on the draft manuscript: Maureen Baikie and Robert Strang, Nova Scotia Health Promotion and Protection; Jayne Boutilier, Teri Cole and Dee Mombourquette, Capital District Health Authority; Li Jin, Health Protection Agency (UK); Jean Rochefort, Institut national de santé publique du Québec; and Graham Tipples, Public Health Agency of Canada.

\section{REFERENCES}

I. Bellini WJ, Sever JL. Measles, mumps, and rubella. In: Specter S, Hodinka RL, Young SA, eds. Clinical virology manual. 3 rd ed. Washington: ASM Press; 2000. p. $501-13$.

2. World Health Organization. Global status of mumps immunization and surveillance. Wkly Epidemiol Rec 2005;48:418-24.

3. Plotkin SA. Mumps vaccine. In: Plotkin SA, Orenstien WA, eds. Vaccines. Philadelphia: Elsevier; 2004. p. 44I-69.

4. Atkinson W, Hamborsky J, McIntyre L, et al., eds.; Centers for Disease Control and Prevention. Epidemiology and prevention of vaccine-preventable diseases: the pink book. gth ed. Washington: Public Health Foundation; 2006.

5. National Advisory Committee on Immunization (NACI), Health Canada. Canadian immunization guide. 6th ed. Ottawa: Canadian Medical Association; 2002. Available: www.phac-aspc.gc.ca/publicat/cig-gci/pdf/cdn_immuniz_guide-2002-6.pdf (accessed $2006 \mathrm{Jul} \mathrm{I} 3$ ).

6. Savage E, White JM, Brown DEW, et al. Mumps epidemic - United Kingdom, 2004-2005. MMWR 2006;55:173-5. Available: www.cdc.gov/mmwr/preview /mmwrhtml/mm5507ar.htm (accessed 2006 Jul I3).

7. Office of the Chief Medical Officer of Health; Nova Scotia Department of Health. Draft Nova Scotia immunization manual. Halifax: The Department; 2006.

8. Office of the Chief Medical Officer of Health; Nova Scotia Department of Health. Notifiable diseases in Nova Scotia: surveillance report 2003-2004. Halifax: The Department; 2005. Available: www.gov.ns.ca/health/downloads/CD_Annual _Report_2003-2004.pdf (accessed 2006 Jul I3).

9. Health Canada. Case definitions for diseases under national surveillance. CCDR 2000;26S3:105. Available: www.phac-aspc.gc.ca/publicat/ccdr-rmtc/oopdf /cdr26s3e.pdf (accessed 2006 Jul I4).

ı. Heymann DL, ed. Control of communicable diseases manual. I8th ed. Washington: APHA [American Public Health Association] Press; 2005.

II. Jin L, Beard S, Brown DW. Genetic heterogeneity of mumps virus in the United Kingdom: identification of two new genotypes. Jnfect Dis 1999;180:829-33.

I2. Vandermeulen C, Roelants $M$, Vermoere $M$, et al. Outbreak of mumps in a vaccinated child population: a question of vaccine failure? Vaccine 2004;22:2713-6.

13. Harling R, White JM, Ramsay ME, et al. The effectiveness of the mumps component of the MMR vaccine: a case control study. Vaccine 2005;23:4070-4.

I4. Peltola H, Davidkin I, Paunio M, et al. Mumps and rubella eliminated from Finland. JAMA 2000;284:2643-7.

15. Jin L, Rima B, Brown C, et al. Proposal for genetic characterization of wild-type mumps strains: preliminary standardization of the nomenclature. Arch Virol 2005; I50:1903-9.

I6. Nojd J, Tecle T, Samuelsson A, et al. Mumps virus neutralizing antibodies do not protect against reinfection with a heterologous mumps virus genotype. Vaccine 200I; I9: I727-3I.

I7. Henry K, Schulte C, Blog D, et al. Mumps outbreak at a summer camp - New York, 2005. MMWR 2006;55:175-7. Available: www.cdc.gov/mmwr/preview /mmwrhtml/mm5507a2.htm (accessed 2006 Jul I3).

I8. Gupta RK, Best J, MacMahon E. Mumps and the UK epidemic 2005. BMJ 2005; 330:1132-5.

I9. Centers for Disease Control and Prevention. Update: multistate outbreak of mumps —United States, January I-May 2, 2006. MMWR 2006;55:559-63. Available: www .cdc.gov/mmwr/preview/mmwrhtml/mm5520a4.htm (accessed 2006 Jul I3).

Correspondence to: Dr. Gaynor Barbara Watson-Creed, Public Health Services, 201 Brownlow Ave., Unit 4, Dartmouth NS B $3 B_{1} W_{2}$; fax 902 481-5803; gaynor.watson-creed@cdha.nshealth.ca 